\title{
Wnt pathway components orient a mitotic spindle in the early Caenorhabditis elegans embryo without requiring gene transcription in the responding cell
}

\author{
Ann Schlesinger, Christopher A. Shelton, Julin N. Maloof, ${ }^{1}$ Marc Meneghini, and Bruce Bowerman ${ }^{2}$ \\ Institute of Molecular Biology, University of Oregon, Eugene, Oregon 97403 USA; ${ }^{1}$ Department of Biochemistry \\ and Biophysics, University of California, San Francisco, California 94143 USA
}

In a four-cell-stage Caenorhabditis elegans embryo, Wnt signaling polarizes an endoderm precursor called EMS. The polarization of this cell orients its mitotic spindle in addition to inducing endodermal fate in one

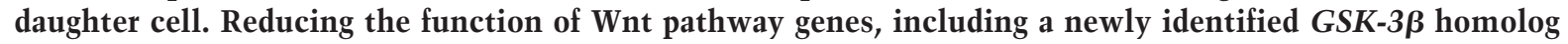
called gsk-3, disrupts endoderm induction, whereas only a subset of these genes is required for proper EMS mitotic spindle orientation. Wnt pathway genes thought to act downstream of gsk-3 appear not to be required for spindle orientation, suggesting that gsk-3 represents a branch point in the control of endoderm induction and spindle orientation. Orientation of the mitotic spindle does not require gene transcription in EMS, suggesting that Wnt signaling may directly target the cytoskeleton in a responding cell.

[Key Words: Cell polarity; endoderm; inductive signaling; mesoderm; cytoskeleton; wingless]

Received February 9, 1999; revised version accepted June 8, 1999.

During asymmetric cell division, correct partitioning of segregated cytoplasmic determinants must be coordinated with the axis of cell division (Lu et al. 1998; Hawkins and Garriga 1999). Such an asymmetric division occurs in the Caenorhabditis elegans embryo when a fourcell-stage embryonic cell, or blastomere, called EMS divides along the anterior/posterior $(\mathrm{A} / \mathrm{P})$ axis. After replication of the EMS centriole, the two centrosomes migrate to align initially along the left/right (L/R) axis (Fig. 1B). Shortly before mitosis, the EMS nucleus and centrosomes rotate $90^{\circ}$. The ensuing mitotic spindle forms along the $\mathrm{A} / \mathrm{P}$ axis, and EMS divides into a posterior daughter, E, that produces endoderm, and an anterior daughter, MS, that makes mesoderm (Sulston et al. 1983; Hyman and White 1987; Fig. 1A).

Positioning of the centrosomes and polarization of endodermal potential within EMS both require signaling from the blastomere posterior to EMS, called $\mathrm{P}_{2}$ (Schierenberg 1987; Goldstein 1992, 1993, 1995a,b; Fig. 1D). The role of $\mathrm{P}_{2}$ signaling was demonstrated using blastomeres isolated from wild-type embryos and cultured in vitro. If EMS is isolated early in its cell cycle and cultured alone, it produces two MS-like daughters that make only mesodermal tissue, such as muscle. Only when $\mathrm{P}_{2}$ is placed in contact with EMS does EMS makes

${ }^{2}$ Corresponding author.

E-MAIL bbowermann@molbio.uoregon.edu; FAX (541) 346-5891. endoderm in addition to mesoderm (Goldstein 1992). Moreover, if EMS is cultured alone, its mitotic spindle elongates along the axis established by centrosome migration, without any rotation (Goldstein 1995b). Contact with $\mathrm{P}_{2}$ early in the EMS cell cycle induces rotation of the EMS nucleus and centrosomes, orienting the mitotic spindle perpendicular to the plane of contact between the two cells (Goldstein 1995b; Fig. 1D). Therefore $\mathrm{P}_{2}$ signaling polarizes EMS such that a posterior daughter is born next to $\mathrm{P}_{2}$ and produces endoderm. EMS remains competent to polarize endoderm potential in response to $\mathrm{P}_{2}$ signaling for longer than it remains competent to orient its mitotic spindle (Goldstein 1992, 1995a,b), suggesting that the signals or their responses may be distinct.

Genetic insight into the nature of $\mathrm{P}_{2}$ signaling has come, in part, from the identification of five mom genes required for endoderm specification. Mothers homozygous for a recessive mutation in any one of these essential genes produce mutant embryos that lack endoderm because both EMS daughters adopt an MS-like fate (Rocheleau et al. 1997; Thorpe et al. 1997). Another maternally expressed gene, pop-1, is required to repress endoderm fate in the anterior EMS daughter, as both EMS daughters in pop-1 mutant embryos adopt an E fate (Lin et al. 1995). The identities of three mom genes and pop-1 are known and all are related to Wnt pathway genes. mom-1 is similar to porcupine (porc), mom-2 to wingless 
A

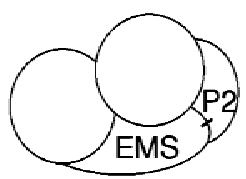

B
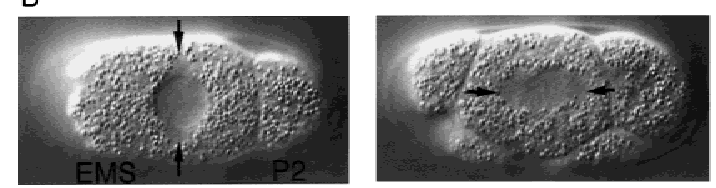

C

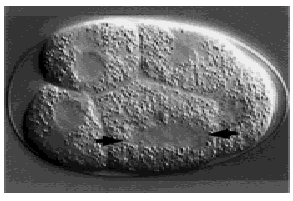

wild-type

D
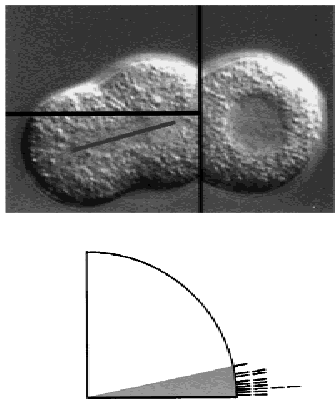

recombined early
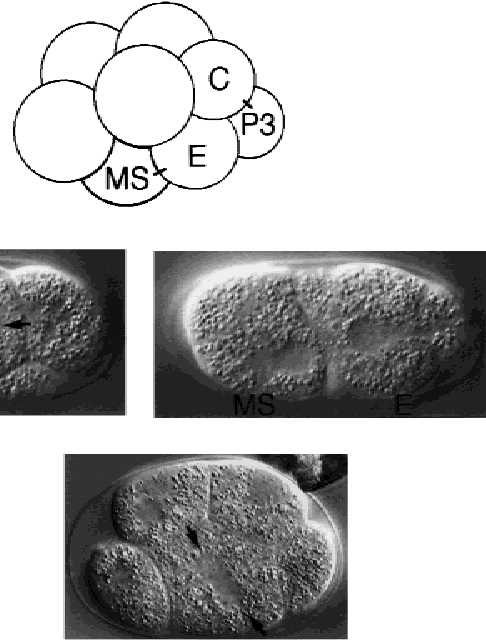

mom-3
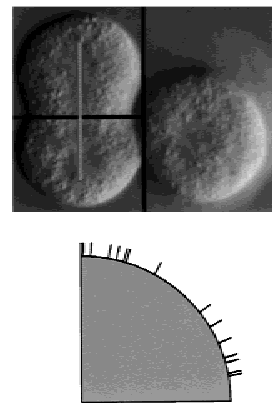

recombined late

Figure 1. Proper EMS mitotic spindle orientation requires signaling from $\mathrm{P}_{2}$. All embryos are shown with anterior to the left. (A) Schematic lateral view of early C. elegans four- and eightcell-stage embryos showing blastomere positions and lineages. $\mathrm{P}_{2}$, the smaller cell, is posterior to its sister EMS. On division, only the posterior EMS daughter, E, is in contact with $\mathrm{P}_{2}$. C and $\mathrm{P}_{3}$ are the daughters of $\mathrm{P}_{2}$. Sister cells are connected by short lines. $(B)$ Nomarski micrograph of a ventral view of a four-cell-stage embryo. EMS centrosomes are indicated by arrows; the centrosomes are visible by their exclusion of cytoplasmic yolk droplets. (Left) The centrosomes oriented along the $\mathrm{L} / \mathrm{R}$ axis after migration; (middle) the centrosomes have rotated $90^{\circ}$ to lie along the $\mathrm{A} / \mathrm{P}$ axis and the EMS spindle has begun to elongate, oriented toward $\mathrm{P}_{2}$; (right) $\mathrm{E}$ and MS after completion of cytokinesis. $(C)$ Lateral views of four-cell-stage embryos. EMS centrosomes are indicated by arrows. (Left) A wild-type embryo with the EMS spindle oriented toward $\mathrm{P}_{2}$; (right) a mom-3 mutant embryo with the EMS spindle incorrectly oriented. $(D)$ Isolated wild-type blastomeres in culture. EMS and $\mathrm{P}_{2}$ blastomeres were separated immediately after $\mathrm{P}_{1}$ division then recombined. $\mathrm{P}_{2}$ is on the right. EMS mitotic spindle angles were measured as described in Material and Methods. (Left) Results from experiments in which $\mathrm{P}_{2}$ and EMS were recombined early during the EMS cell cycle while EMS was competent to respond to the $\mathrm{P}_{2}$ signal. All spindles in these experiments oriented correctly, with spindle angles between $1^{\circ}$ and $12^{\circ}$. (Right) Results from experiments in which $\mathrm{P}_{2}$ and EMS were recombined after EMS could no longer respond to the $\mathrm{P}_{2}$ signal. The range of spindle angles from $10^{\circ}$ to $90^{\circ}$ indicates that EMS spindles in these experiments were randomly oriented with respect to $\mathrm{P}_{2}$.

$(w g)$, and mom-5 to frizzled $(f z)$ (Rocheleau et al. 1997; Thorpe et al. 1997). pop-1 is related to Drosophila pangolin/dTCF (pan) and mammalian TCF/LEF family members that encode putative transcription factors regulated by Wnt signaling (Lin et al. 1995, 1998). Therefore, the widely conserved Wnt signal transduction pathway is required for endoderm induction during embryogenesis in C. elegans (for review, see Lu et al. 1998; Hawkins and Garriga 1999).

To determine whether Wnt signaling regulates mitotic spindle orientation in addition to polarizing endoderm potential in EMS, we asked if rotation of the EMS nucleus and its associated centrosomes requires the mom and other Wnt pathway genes. Previous studies have documented a mitotic spindle orientation defect in an eight-cell-stage blastomere called ABar in mom mutant embryos, as well as extensive defects in mitotic spindle orientation and blastomere positioning in mom1;mom-2 double mutants (Rocheleau et al. 1997; Thorpe at al. 1997). EMS spindle orientation has not been examined in mutant embryos lacking the function of individual mom genes. Using blastomeres isolated from mutant embryos and cultured in vitro, we show that a subset of the C. elegans Wnt pathway genes known to be required for endoderm specification also are required for proper orientation of the EMS spindle. In addition, we have identified a $C$. elegans $G S K-3 \beta$ gene that may function as a branchpoint for endoderm specification and spindle orientation. Finally, we present evidence that the induced rotation of the EMS mitotic spindle does not require gene transcription, suggesting that Wnt signaling may target the cytoskeleton directly in a responding cell.

\section{Results}

Wnt pathway components mom- $1 /$ porc and mom-5/fz, but not mom-2/wg, are required for proper spindle orientation

Both endoderm specification and proper EMS spindle orientation require signaling from $\mathrm{P}_{2}$ (see introductory section). To determine if the mom genes, which are required for endoderm induction, also are required for rotation of the EMS nucleus and its pair of centrosomes, we first observed the orientation of the EMS spindle in developing mutant embryos using Nomarski optics. In wild-type embryos, the EMS centrosomes initially align along the $\mathrm{L} / \mathrm{R}$ axis of the embryo. Before nuclear envelope break- 
down, the EMS nucleus/centrosome complex rotates to form a spindle along the $\mathrm{A} / \mathrm{P}$ axis (Hyman and White 1987; Fig. 1B). The spindle then elongates, with only the posterior daughter of EMS born in contact with $\mathrm{P}_{2}$. In mom-1/porc, mom-3, and mom- $5 / f_{z}$ mutant embryos, we observed defects in EMS spindle orientation. The axis of orientation varied, exhibiting significant dorsal/ventral or L/R components (Fig. 1C; data not shown). As the mitotic spindles elongated, however, they invariably assumed a mostly A/P orientation before the end of cytokinesis (data not shown). The A/P axis of EMS also is its longest axis, and therefore spindles may rotate passively to accommodate their length, making it somewhat difficult to quantitate this phenotype when observing intact embryos within their chitinous eggshells.

To facilitate scoring EMS mitotic spindle orientation in wild-type and mutant embryos, we isolated blastomeres in culture medium after removing the eggshells (Goldstein 1995b; see Materials and Methods). We isolated the smaller, posterior-most blastomere in a twocell-stage embryo, called $\mathrm{P}_{1}$, and allowed it to divide into its daughters, $\mathrm{P}_{2}$ and EMS. The orientation of the mitotic spindle in EMS was then measured relative to the plane of contact between EMS and $\mathrm{P}_{2}$. As illustrated in Figure 2 , the range of wild-type EMS spindle angles is within $19^{\prime}$ of a line perpendicular to the plane of contact with $\mathrm{P}_{2}$. The mitotic spindles in EMS blastomeres from mom$1 /$ porc, mom-3, and mom-5/fz mutant embryos, however, generated a much wider range of angles, showing nearly random orientations (Fig. 2). The EMS spindle in mom-2/wg mutant blastomeres oriented more normally (Fig. 2). mom-4 experiments yielded a wide range of EMS spindle angles $\left(0^{\circ}-90^{\circ}\right)$. Only 4/26 mom-4 EMS spindles were abnormal, however, whereas the majority oriented properly. Therefore mom-1/porc, mom-5/fz, mom-3, and perhaps to a lesser degree mom- 4 are required for both endoderm induction and proper EMS spindle orientation, but mom-2/wg appears to be required more specifically for endoderm induction.

\section{A subset of Wnt pathway components are required for EMS spindle orientation}

Because mom-1/porc and $m o m-5 / f z$ are required for EMS spindle orientation, we examined mutant blastomeres lacking the function of other Wnt pathway components required for polarizing endoderm potential in EMS, as well as blastomeres lacking the function of a $C$. elegans GSK-3 $\beta$ gene that we identified using degenerate PCR (Fig. 3; Materials and Methods). The predicted GSK-3 protein is $76 \%$ similar and $69 \%$ identical to the GSK-3 $\beta$ encoded by Drosophila zeste white 3 (zw3). We investigated the function of gsk-3 using RNA-mediated interference (RNAi), a method that involves microinjecting double-stranded exonic RNA into the syncytial nematode ovary to reduce the function of the corresponding maternally expressed gene (Fire et al. 1998; see Materials and Methods). We refer to embryos from mothers microinjected with gsk-3 RNA as gsk-3(RNAi) embryos. After isolation of gsk-3(RNAi) $\mathrm{P}_{1}$ blastomeres, we observed that EMS spindles were randomly oriented (Fig. $2)$. We conclude that, in addition to mom-1/porc and mom- $5 / f z$, the Wnt pathway component gsk-3 also is required to orient the EMS mitotic spindle.

GSK-3 $\beta$ is thought to interact with both $\beta$-catenin/ Armadillo (Arm) and adenomatous polyposis ecoli (APC), possibly phosphorylating one or both proteins (Rubinfeld et al. 1996; Miller and Moon 1997). C. elegans
Figure 2. A subset of identified Wnt pathway genes are required for proper orientation of the EMS spindle. Isolated $\mathrm{P}_{1}$ blastomeres from different mutant strains were cultured in vitro; on $\mathrm{P}_{1}$ division, $\mathrm{P}_{2}$ and EMS were left in contact and the angle of the EMS spindle was measured. Spindle angles were scored and recorded as described in Materials and Methods. The left-most quadrant shows wild-type control data, with spindle angles ranging from $0^{\circ}$ to $19^{\circ}$. The range of spindle angles in EMS blastomeres from embryos mutant for mom-1/porc $\left(1^{\circ}-88^{\circ}\right)$, mom- $5 / f z$ $\left(0^{\circ}-90^{\circ}\right)$, mom-3 $\left(1^{\circ}-90^{\circ}\right)$, and gsk $-3\left(1^{\circ}-90^{\circ}\right)$ are wider than wild-type, indicating a lack of orientation with respect to the plane of contact with $\mathrm{P}_{2}$. The defects in EMS may repre-

sent a randomization of spindle orientation, or an incompletely penetrant failure to rotate the nucleus/centrosome complex. In some cases, endoderm induction occurred in the absence of correct EMS spindle orientation, and vice versa. Examples of such situations have been indicated for mom-1/porc, mom-3, and mom-5/fz. A $(+)$ next to a spindle angle mark indicates the production of endoderm in spite of abnormal EMS spindle orientation and a (-) indicates the lack of endoderm in some partial embryos with normal EMS spindle angles. mom- 4 experiments yielded a wide range of EMS spindle angles $\left(0^{\circ}-90^{\circ}\right)$. Only $4 / 26$ were abnormal, whereas the majority of spindles oriented properly. A one-sided Student $t$-test comparing wild-type with mom- 4 yields a $P$-value of 0.18 , indicating that the mom- 4 data set is not significantly different from wild type. Spindle angle ranges for $m o m-2 / \mathrm{wg}^{\circ}\left(0^{\circ}-28^{\circ}\right)$, apr-1/APC $\left(3^{\circ}-34^{\circ}\right)$, wrm-1/arm $\left(1^{\circ}-17^{\circ}\right)$, pop-1/pan $\left(1^{\circ}-32^{\circ}\right)$, and the apr-1;wrm- 1 double $\left(0^{\circ}-13^{\circ}\right)$ were similar to wild type. Mammalian and Drosophila genes names are aligned below their C. elegans homologs. mom-3 and mom-4 have not yet been cloned. 
A

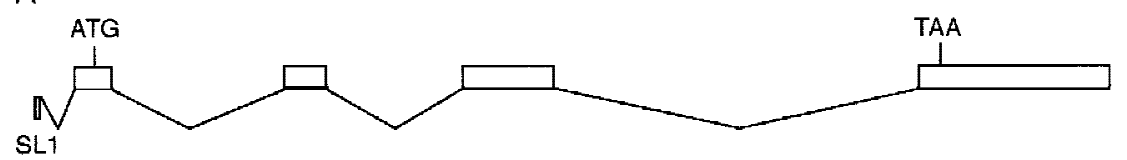

B

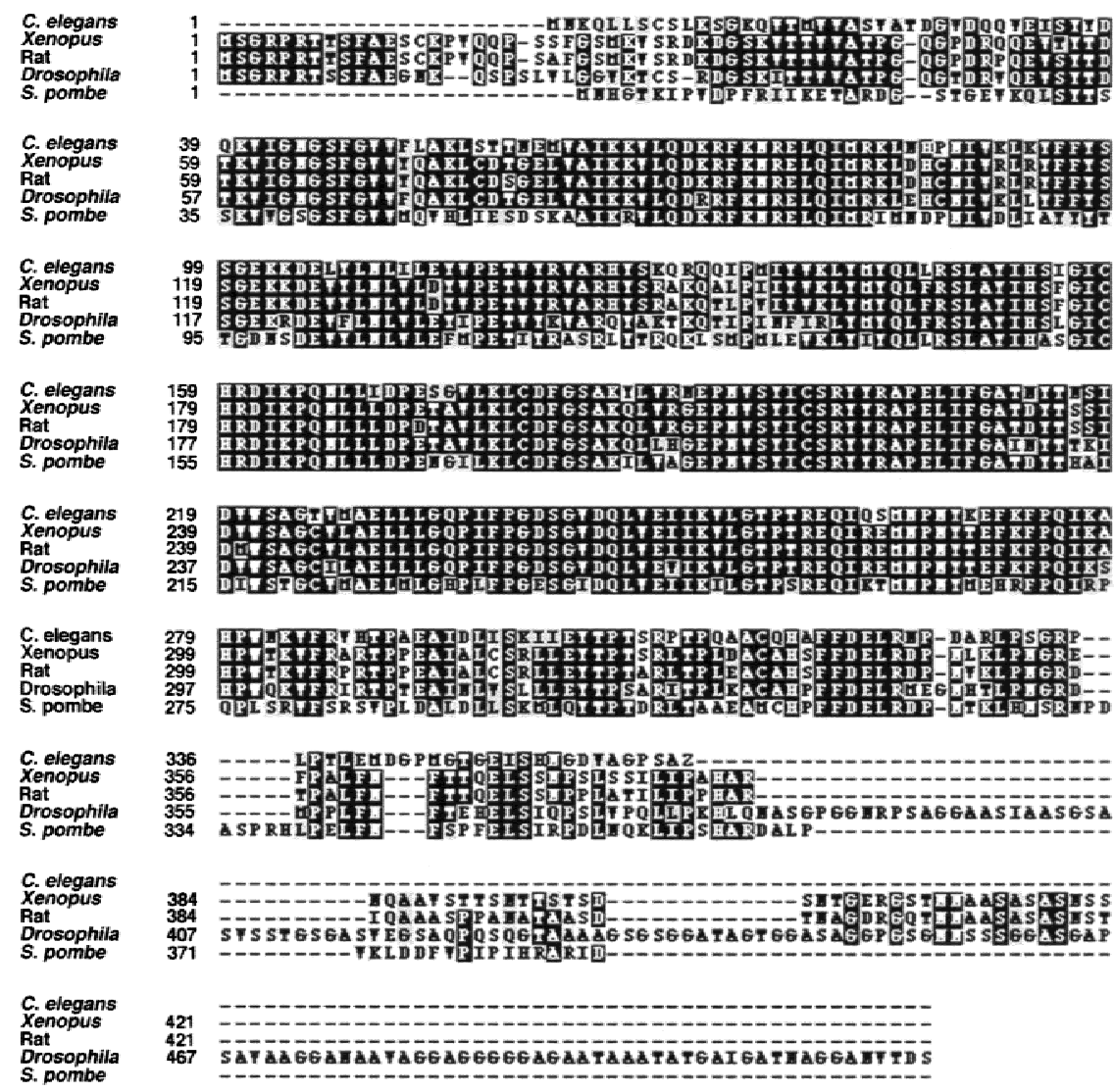

Figure 3. gsk-3 is a C. elegans GSK-3 $\beta$ homolog. $(A)$ Gene structure of $C$. elegans gsk-3 predicted by comparing the cDNA sequence with genomic sequence of clone Y18D10. (B) Alignment of the predicted $C$. elegans GSK-3 sequence with GSK-3 proteins from other organisms. C. elegans GSK-3 is $78 \%$ similar (73\% identical) with Xenopus GSK-3, $77 \%$ similar $172 \%$ identical) with Rat, $76 \%$ similar $(69 \%$ identical) with Drosophila, and 68\% similar (59\% identical) with Schizosaccharomyces pombe. genes related to $\beta$-catenin/arm and APC, called Wormadillo-1 (wrm-1) and APC-related-1 (apr-1), are known to be required for endoderm induction (Rocheleau et al. 1997). Reducing the function of either wrm-1/arm or apr-1/APC, using RNAi, does not significantly affect EMS mitotic spindle orientation (Fig. 2). To test for any possible functional redundancy between wrm-1/arm and apr-1/APC, both of which contain multiple arm repeats (Rocheleau et al. 1997), RNAs from both genes were injected simultaneously. The EMS spindle in these double mutants oriented correctly, further indicating that wrm1/arm and apr-1/APC are not required (Fig. 2). Although RNAi may not completely eliminate gene function in these experiments, the lack of requirement for wrm-1/ arm is particularly compelling, as reducing wrm-1/arm function by RNAi causes a fully penetrant loss of endoderm (Rocheleau et al. 1997; A. Schlesinger et al., unpubl.). In contrast, incorrectly oriented spindles were observed in $57 \%$ mom $-5 / f z$ mutant embryos, even though only $5 \%$ of $\mathrm{mom}-5 / f z$ mutants lack endoderm (Fig. 2; Rocheleau et al. 1997; Thorpe et al. 1997). Finally, EMS spindles are oriented normally in mutant embryos lacking maternal expression of pop-1/pan (Fig. 2), which encodes an HMG-domain protein targeted by Wnt signaling during endoderm induction (Lin et al. 1995, 1998; Rocheleau et al. 1997; Thorpe et al. 1997). Therefore, the genes acting downstream of gsk-3 in the Wnt pathway are not required for proper EMS spindle orientation even when both wrm-1/arm and apr-1/APC functions are compromised simultaneously.

The Wnt pathway in EMS may branch at gsk-3 to regulate both endoderm specification and mitotic spindle orientation

Next we examined the role of gsk-3 during endoderm specification. Most gsk-3(RNAi) embryos produce intestine $(82 \%$; Table 1). Approximately one-fifth lack endoderm and instead make large clumps of cells similar in appearance to the extra pharyngeal tissue observed in mom mutant embryos (Table 1; data not shown). To determine the origins of the endoderm made in gsk- 
Table 1. Cell fate in gsk-3(RNAi) embryos

A. Polarizing optics ${ }^{\mathrm{a}}$

Percent producing
endoderm (total)

B. Tissue production ${ }^{\mathrm{b}}$

\begin{tabular}{lcc} 
& $\begin{array}{c}\text { Percent making } \\
\text { pharyngeal } \\
\text { muscle }\end{array}$ & $\begin{array}{c}\text { Percent making } \\
\text { body wall } \\
\text { muscle }\end{array}$ \\
\hline $\begin{array}{l}\text { Wild type isolated E } \\
\text { gsk-3(RNAi) isolated E }\end{array}$ & $0(0 / 9)$ & 0 \\
\hline
\end{tabular}

a Using polarizing optics to detect the presence of rhabditin granules, endoderm was scored in terminally differentiated embryos following ablation of indicated blastomeres. The total number of ablations scored is indicated in parenthesis.

${ }^{\mathrm{b}}$ Tissue production was scored using tissue-specific antibodies on terminally differentiated, fixed embryos following ablation of indicated blastomeres. Embryos were stained with 3NB12, a monoclonal antibody that recognizes pharyngeal muscle cells, or 5.6, one that recognizes body wall muscle. An asterisk indicates that all positive staining occurred only in embryos that did not make endoderm.

3(RNAi) embryos, we used a laser microbeam to kill all but one early blastomere, subsequently scoring the ability of the isolated blastomere to produce endodermal or mesodermal cell types (Table 1). Whereas E always makes endoderm in wild-type embryos, $44 \%$ of gsk3(RNAi) E blastomeres failed to make endoderm and instead produced pharyngeal and body wall muscle cells, consistent with E adopting an MS-like fate (Table 1). Therefore $g s k-3$ is required positively for the specification of endoderm in C. elegans. As genes thought to act downstream of $g s k-3$ are required only for endoderm specification, whereas gsk-3 itself is required for both endoderm specification and EMS spindle orientation, we conclude that gsk-3 represents a branchpoint by which Wnt signaling regulates both processes.

The production of endoderm by $\mathrm{E}$ in only $56 \%$ of $g s k$ 3(RNAi) embryos does not account for the observation that $82 \%$ of intact mutant embryos produce endoderm. Accounting for this discrepancy, we found that a dorsalposterior eight-cell-stage descendant of $\mathrm{P}_{2}$ called C ectopically makes endoderm in most gsk-3(RNAi) embryos. These results indicate that in wild-type embryos gsk-3 negatively regulates endoderm production in $\mathrm{C}$ while positively specifying endoderm in EMS (see Discussion).
EMS spindle orientation requires mom-1/porc and mom-3 in $P_{2}$, and mom-5/fz and gsk-3 in EMS

Having established a requirement for mom-1/porc, mom-3, mom-5/fz and gsk-3 in spindle orientation, we constructed genetic chimeras to determine if these and other genes are required in $\mathrm{P}_{2}$ to signal, or in EMS to respond to signaling. We isolated blastomeres from mutant and wild-type embryos, and in each experiment reassociated a mutant cell with a wild-type partner (see Materials and Methods). When placed in contact with a wild-type EMS, mom-1/porc, mom-2/wg, and mom-3 mutant $\mathrm{P}_{2}$ blastomeres failed to properly orient EMS mitotic spindles, whereas EMS cells from these mutant embryos were capable of responding to wild-type $\mathrm{P}_{2}$ cells (Fig. 4). EMS blastomeres from mom-5/fz and $g s k-3 \mathrm{mu}-$ tants did not respond competently to wild-type $\mathrm{P}_{2}$ signaling, but mom-5/fz and gsk-3 mutant $\mathrm{P}_{2}$ blastomeres properly oriented the spindle of wild-type EMS cells (Fig. 4). Orientation of the EMS spindle was normal in the mom-4 mosaics. Therefore, if mom-4 is required for spindle orientation (Fig. 2), apparently either blastomere can provide the necessary function, as one cell is wildtype in the chimeras (Fig. 4).

The requirement for mom-2/Wg in $\mathrm{P}_{2}$ signaling in genetically mosaic partial embryos was surprising (Fig. 4), as mom-2/Wg is not required for EMS mitotic spindle orientation after isolating $\mathrm{P}_{1}$ blastomeres from mom-2/ wg mutant embryos (Fig. 2). When assembling mosaic partial embryos in culture, however, we did not strictly control the age of $\mathrm{P}_{2}$ when placed in contact with EMS (see Materials and Methods), as wild-type $\mathrm{P}_{2}$ blastomeres remain competent to signal throughout their cell cycle (Goldstein 1995b; A. Schlesinger et al., unpubl.). Although the age of a wild-type $\mathrm{P}_{2}$ does not affect its ability to orient an EMS mitotic spindle, mom-2/wg mutant $\mathrm{P}_{2}$ blastomeres are able to signal only early in the $\mathrm{P}_{2}$ cell cycle. When assembled into a genetic chimera within the first five minutes of their cell cycle, mom-2/wg mutant $\mathrm{P}_{2}$ blastomeres properly orient the mitotic spindle of a wild-type EMS; however, older $\mathrm{P}_{2}$ blastomeres fail to do so (Fig. 4B). Therefore, mom-2/wg mutant $\mathrm{P}_{2}$ blastomeres prematurely lose their signaling ability. This defect in signaling from older $\mathrm{P}_{2}$ blastomeres is specific for mom-2/wg mutant embryos and does not occur in genetic mosaic experiments with mom-1/porc or mom-3 mutant $\mathrm{P}_{2}$ blastomeres (data not shown). We conclude either that our mom-2/wg mutant alleles retain some maternal function that rapidly fades with time, or that other signals act in parallel with $\mathrm{mom}-2 / \mathrm{wg}$ to polarize EMS before cell division.

\section{$P_{2}$ signaling orients the mitotic spindle in EMS independent of gene transcription}

The induced rotation of the EMS nucleus and centrosomes involves a polarization of the cytoskeleton. $\mathrm{P}_{2}$ signaling could polarize the cytoskeleton in EMS either directly, or as a secondary consequence of regulated gene 
A
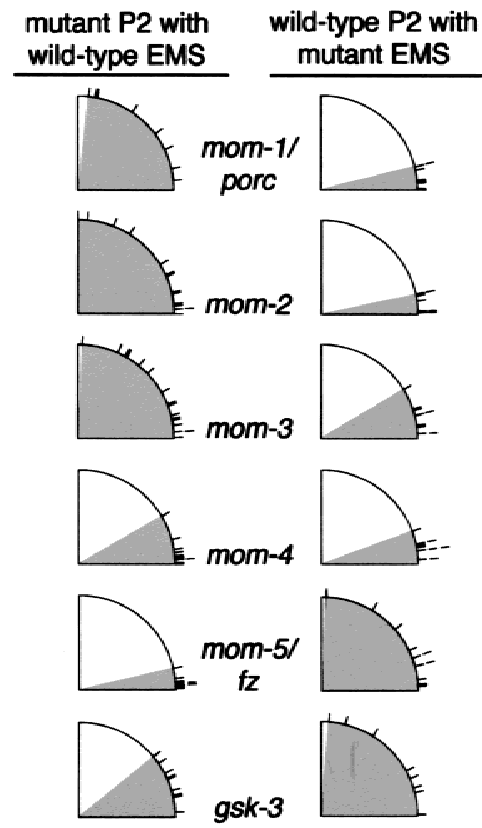

B

'old' mom-2 P2 'young' mom-2 P2
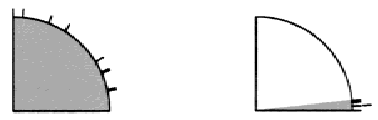

Figure 4. mom-1/porc and mom-3 are required in $\mathrm{P}_{2}$ and mom$5 / f z$ and $g s k-3$ in EMS for spindle orientation. (A) Mutant and wild-type EMS and $\mathrm{P}_{2}$ blastomeres were isolated and then recombined to form genetic chimeras (Materials and Methods). (Left column) Schematic summaries of EMS spindle angle data from experiments with a mutant $\mathrm{P}_{2}$ blastomere recombined with a wild-type EMS. For these experiments, EMS spindle ranges were as follows: mom-1/porc, $6^{\circ}-83^{\circ}$; mom- $2 / \mathrm{wg}, 0^{\circ}-$ $90^{\circ}$; mom- $3,1^{\circ}-87^{\circ}$; mom $-4,0^{\circ}-30^{\circ} ;$ mom- $5 / f z, 1^{\circ}-13^{\circ}$; gsk- 3 , $3^{\circ}-39^{\circ}$. (Right column) Data from experiments in which a wildtype $\mathrm{P}_{2}$ was recombined with a mutant EMS. Spindle angle ranges for these experiments are mom-1/porc, $0^{\circ}-14^{\circ}$; mom-2/ wg, $1^{\circ}-12^{\circ}$; mom $-3,3^{\circ}-31^{\circ}$; mom $-4,3^{\circ}-20^{\circ}$; mom $-5 / f z, 4^{\circ}-87^{\circ}$; gsk-3, $1^{\circ}-85^{\circ}$. (B) Genetic chimeras made with wild-type EMS and mom-2/wg mutant $\mathrm{P}_{2}$. (Right) A 'young' EMS was placed into contact with an 'old' mom-2/wg mutant $\mathrm{P}_{2}$ isolated from an older embryo such that $\mathrm{P}_{2}$ divided before EMS (range of spindle angles, $12^{\circ}-90^{\circ}$ ). (Left) A 'young' EMS was placed into contact with a mom-2/Wg mutant $\mathrm{P}_{2}$ of similar age (range of spindle angles: $0^{\circ}-6^{\circ}$ ). For definition of young and old blastomeres, see text.

transcription in EMS. Because some components of the Wnt pathway in Drosophila are required for the proper orientation of epidermal bristles, and bristle orientation depends on the proper organization of the actin cytoskeleton, it has been long suspected that Wnt signaling might directly target the cytoskeleton (Wong and Adler 1993; see Discussion). Determining if the signal that induces endoderm in C. elegans primarily targets the cytoskeleton or the regulation of gene expression is difficult, as endoderm markers appear only after several subsequent developmental steps that clearly involve gene transcription. The EMS mitotic spindle rotation occurs within minutes of the $\mathrm{P}_{2}$ signal, however, and we have therefore been able to test if this response to Wnt signaling requires gene transcription in the responding cell.

Using cultured isolated blastomeres, we quantitatively assayed the induced rotation of the EMS centrosome/nucleus complex after blocking transcription using a chemical inhibitor and RNA interference. To provide an internal control for monitoring gene transcription, we used a transgenic C. elegans strain that expresses the green fluorescent protein (GFP) under control of the pes-10 promoter (Fig. 5A). The pes-10 gene is among the earliest expressed zygotic genes that have been identified in C. elegans, with pes-10 RNA detectable in EMS and its descendants beginning at the fourcell stage (Seydoux and Fire 1994; Seydoux et al. 1996). Culturing blastomeres from pes-10::GFP embryos in actinomycin D, a chemical inhibitor of transcription, blocks detectable expression of GFP, as does RNA-mediated gene interference with ama-1 RNA /see Materials and Methods). The ama-1 gene encodes the largest subunit of RNA polymerase II (Pol II), and RNAi has been used previously to block its function (Powell-Coffman et al. 1996). In our most rigorous tests, we cultured blastomeres from ama-1(RNAi)/pes-10::GFP embryos in actinomycin D. Detectable GFP expression was absent, but in all cases EMS mitotic spindle orientation was normal (Fig. 5B). Previous studies have also suggested that cleavage patterns in early C. elegans embryos appear normal after blocking transcription (Edgar et al. 1994; PowellCoffman et al. 1996). We conclude that components of the Wnt signal transduction pathway control EMS

A

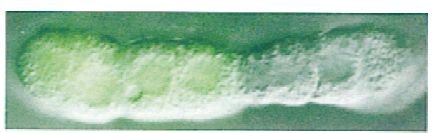

B

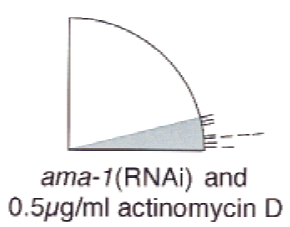

Figure 5. Transcriptional inhibitors do not block proper EMS spindle orientation. (A) A $\mathrm{P}_{1}$ blastomere from pes-10::GFP strain was isolated and cultured until the equivalent of the 28-cell stage. The fluorescence image was superimposed on a Nomarski micrograph. GFP fluorescence is visible in the descendants of EMS but not in the $\mathrm{P}_{2}$-derived lineages. $(B)$ Embryos were isolated from pes-10::GFP hermaphrodites injected with ama-1 RNA. $\mathrm{P}_{1}$ blastomeres from these embryos were cultured in media containing $0.5 \mu \mathrm{g} / \mathrm{ml}$ actinomycin D. EMS was allowed to divide and the EMS spindle angle was recorded. In all cases, although GFP fluorescence was never detected, EMS mitotic spindles oriented properly with respect to the plane of contact with $\mathrm{P}_{2}$. Correct EMS orientation was also seen in EMS cells derived from ama-1(RNAi) $\mathrm{P}_{1}$ blastomeres cultured in higher concentrations of actinomycin $\mathrm{D}(10-20 \mu \mathrm{g} / \mathrm{ml}, n=6 / 6$; see Materials and Methods). 
spindle rotation by directly targeting the cytoskeleton in EMS, not by regulating gene expression.

\section{Discussion}

The polarization of endoderm potential in EMS and the orientation of its mitotic spindle both require Wnt pathway components

Our analysis indicates that Wnt signaling from $\mathrm{P}_{2}$ coordinates the polarization of endoderm potential and orientation of the EMS mitotic spindle during C. elegans embryogenesis. Having both outcomes depend on shared genetic components perhaps ensures that differentially localized cytoplasmic determinants are appropriately segregated into the two daughters on cell division. Nevertheless, the two responses in EMS are separable-in some mom mutants, proper spindle orientation can occur in the absence of endoderm induction (Fig. 2). Moreover, endoderm can be induced during most of the EMS cell cycle, whereas its spindle can be oriented in response to $\mathrm{P}_{2}$ signaling only if contact between the two blastomeres is made early in the EMS cell cycle /Goldstein 1995a,b). These differences in the two EMS responses may be due to $\mathrm{P}_{2}$ expressing more than one signal, or perhaps to differences in the response pathways beyond a gsk-3 branchpoint (see below). The temporally restricted ability of mom-2/wg $\mathrm{P}_{2}$ blastomeres to properly orient EMS spindles (Fig. 4B) may reflect the presence of separate, functionally redundant signals. An unidentified signal for spindle orientation may be expressed by $\mathrm{P}_{2}$ early in its cell cycle, masking any requirement for mom-2/wg.

\section{C. elegans gsk-3 is a branchpoint for polarizing endoderm potential and orienting the EMS mitotic spindle}

We have shown that gsk-3 and all upstream Wnt pathway components so far identified in our system, with the possible exception of $m o m-2 / w g$, are required for both endoderm polarization and for mitotic spindle orientation in EMS. In contrast, the identified nematode Wnt pathway components predicted to act downstream of gsk-3 (apr-1/APC, wrm-1/arm, and pop-1/pan) are required for endoderm induction but not for spindle orientation. We therefore propose a branchpoint at gsk-3, to explain how Wnt signaling from $\mathrm{P}_{2}$ regulates the asymmetric division of EMS (Fig. 6). Pathway components beyond $g s k-3$ that act in the spindle orientation branch remain unknown, although several genes with more general roles in spindle orientation during C. elegans embryogenesis have been identified (for review, see Kemphues and Strome 1997). Recent studies indicate that Wnt signaling in Drosophila also branches within responding cells to regulate both fate specification and tissue polarity (Axelrod et al. 1998; Boutros et al. 1998). This branchpoint occurs at dishevelled (dsh), however, before $z w 3 / G S K-3 \beta$. Therefore the induction of multiple
A

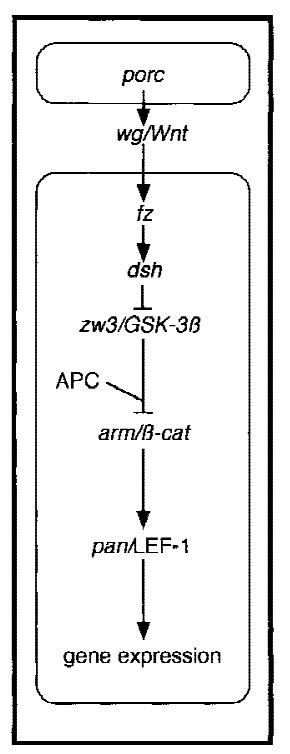

$\mathrm{B}$

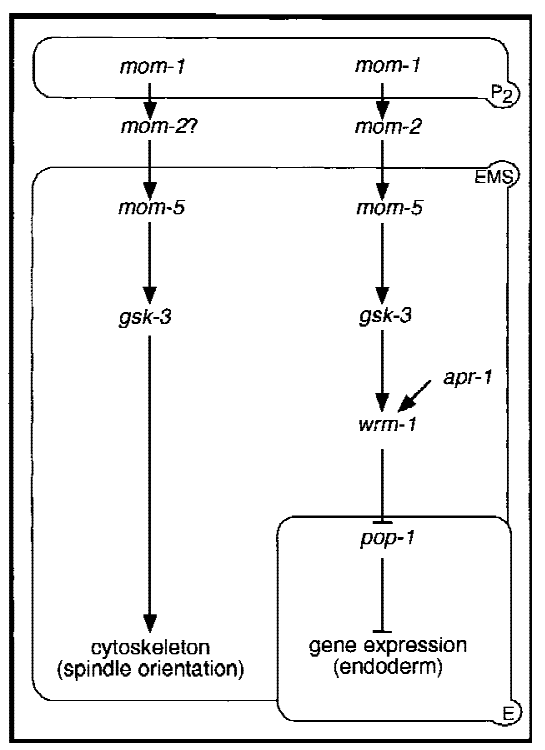

Figure 6. Model for Wnt signaling in the early C. elegans embryo. The Wnt pathway in the early $C$. elegans embryo $(B)$ is contrasted to the 'canonical' pathway derived from experimental data from other organisms ( $A$; see Cadigan and Nusse 1997). In this diagram, the signaling cell is drawn above the responding cell. As shown in $B$, Wnt signaling is required for both EMS mitotic spindle orientation (left) and endoderm specification (right), although there may be separate signaling ligands for these two processes (Rocheleau et al. 1997; Thorpe et al. 1997; A. Schlesinger et al., unpubl.). gsk-3 may represent a branchpoint in the EMS Wnt pathway, regulating both endoderm induction and mitotic spindle orientation. Not depicted but also possible are arrows from gsk-3 directly to the EMS spindle, and directly to the cytoskeleton during endoderm induction. In contrast to the roles of pan and LEF-1, which are converted into activators by Wnt signaling, pop-1 acts purely as a repressor in the C. elegans pathway (Cadigan and Nusse 1997). Release from POP-1-mediated repression of endoderm occurs not in EMS but in its posterior daughter, $\mathrm{E}$.

responses by Wnt signaling may involve branches within responding cells that execute distinct outputs by varying downstream effectors.

\section{gsk-3 acts positively to promote both endoderm specification and proper mitotic spindle orientation}

We have shown that the C. elegans GSK-3 $\beta$ homolog, gsk-3, acts positively during both endoderm specification and mitotic spindle orientation in EMS. This positive role contrasts with the negative roles for GSK-3 $\beta$ during Wnt signal transduction as documented in Drosophila, Xenopus, and humans (Boyle et al. 1991; Siegfried et al. 1992; He et al. 1995). In these studies of the Wnt pathway, GSK-3 $\beta$ is thought to be active in the absence of Wnt signaling, promoting the degradation of $\beta$-catenin in a complex with APC and other proteins (Rubenfeld et al. 1996; Ikeda et al. 1998). In this canonical view, Wnt signaling blocks GSK-3 $\beta$ activity, thereby sta- 
bilizing $\beta$-catenin, which subsequently can translocate to the nucleus and activate target gene expression by binding to and converting ubiquitous HMG domain repressors into transcriptional activators (Brannon et al. 1997; Fig. 6).

The response to Wnt signaling during endoderm induction in C. elegans appears to differ substantially from the canonical model. Instead of converting a repressor into a transcriptional activator, Wnt signaling from $\mathrm{P}_{2}$ down-regulates the HMG domain protein POP-1 in the posterior daughter of the responding cell, thereby promoting endodermal fate (Rocheleau et al. 1997; Thorpe et al. 1997; Fig. 6). To reconcile these different observations, we suggest that Wnt signaling may induce the coincident degradation of POP-1/PAN and WRM-1/arm in E but not MS. This model invokes the established abilities of $\beta$-catenin/arm to form a complex with HMG domain proteins, and of GSK-3 $\beta$ to promote the degradation of $\beta$-catenin. How Wnt signaling could be adapted to activate, instead of block, GSK- $3 \beta$ function in this pathway remains entirely unclear, as does the ability of GSK-3 to positively promote mitotic spindle rotation, which occurs independently of pop-1/pan function. Our understanding of these issues may improve once the subcellular localizations of different Wnt pathway components are determined, and more interacting factors are identified.

Because all Wnt pathway components are required positively for endoderm induction in EMS, it has not been possible to order the function of different gene products relative to each other using double mutant analyses. Nevertheless, other data suggest that components of the Wnt pathway function in the same relative order during endoderm induction as they do in the canonical pathway. First, the excess endoderm phenotype of pop-1/pan mutants is epistatic to the mom-2/wg, mom-5/fz, wrm1/arm, gsk-3, and mom-4 mutant phenotypes, indicating that pop-1/pan acts downstream of these genes (Rocheleau et al. 1997; Thorpe et al. 1997; data not shown). Second, our analysis of genetically mosaic partial embryos shows that mom-5/fz and gsk-3 are both required in EMS, the responding cell, and are therefore downstream of $m o m-1 /$ porc and $m o m-2 / w g$, genes that are required in the signaling cell, $\mathrm{P}_{2}$. Third, we note that all Wnt pathway components up to and including gsk-3, except possibly mom-2/wg, are required both to polarize endoderm potential and to orient the EMS mitotic spindle, whereas all Wnt pathway components downstream of $g s k-3$ are required only for endoderm induction. This latter observation is consistent with gsk-3 functioning upstream of wrm-1/arm and apr-1/APC. Given these similarities to the canonical Wnt pathway, it seems likely that the relative order in which Wnt pathway components function is conserved during endoderm induction in C. elegans in spite of their different regulatory relationships.

Finally, our finding that gsk-3 acts positively during endoderm induction is not without precedent for a GSK$3 \beta$ family member. A GSK-3 $\beta$ homolog in Saccharomyces cerevisiae, Rim $11 \mathrm{p}$, positively regulates the expres- sion of meiotic genes during sporulation by complexing with and phosphorylating two transcription factors, Ume6p and Ime1p (Mandel et al. 1994; Strich et al. 1994; Rubin-Bejerano et al. 1996; Malathi et al. 1997). Furthermore, we note that gsk-3 in C. elegans appears to be required both positively and negatively to regulate the specification of endoderm in the early embryo. Whereas E fails to produce endoderm in fewer than half of gsk-3 mutant embryos, a daughter of $\mathrm{P}_{2}$ called $\mathrm{C}$ produces endoderm ectopically in approximately three-fourths of the mutant embryos (Table 1). Therefore, gsk-3 is required to negatively regulate endoderm specification in $\mathrm{P}_{2}$, the sister of EMS. These results suggest that gsk-3 function is capable of generating opposite outputs even within a single organism, perhaps through the intervention of other regulatory pathways.

\section{The cytoskeleton as the primary target of cell signaling}

Because rotational positioning of the EMS centrosomes appears to not require gene transcription, we suggest that $\mathrm{P}_{2}$ to EMS signaling directly targets the cytoskeleton in EMS. Microtubules are the primary structural component of the mitotic spindle and are thought to interact with cortical microfilaments during rotation of the nucleus/centrosome complex in EMS (Hyman 1989; Goldstein et al. 1993; Waddle et al. 1994). Either microtubules or microfilaments could be targets of Wnt signaling, as both are required for correct orientation of the mitotic spindle in EMS (Hyman and White 1987; Goldstein 1995b). Wnt pathway genes have been implicated in cytoskeleton-related processes in other systems. Mammalian GSK-3 $\beta$ can phosphorylate the microtubule-associated protein tau, and this activity is regulated by $d s h$, another Wnt pathway component (Wagner et al. 1997). Therefore $P_{2}$ signaling might influence microtubule dynamics directly through gsk-3. Alternatively, $\mathrm{P}_{2}$ signaling might influence spindle orientation in EMS by locally activating microtubule motor complexes that directly interact with astral microtubules to orient the nucleus/centrosome complex before cell division.

It seems likely that Wnt signaling directly targets the cytoskeleton to control other developmental processes. For example, the polarized organization of hair cells in the Drosophila epithelium appears to be controlled by the orientation of actin microfilaments, which in turn are regulated by tissue polarity genes including $f z$ and dsh (Wong and Adler 1993; Axelrod et al. 1998; Boutros et al. 1998). Wnt signaling in C. elegans may also target the cytoskeleton to polarize endoderm potential in EMS, as the polarization of endoderm potential appears to require functional microfilaments and microtubules (Goldstein 1995a). Alternatively, $\mathrm{P}_{2}$ signaling may simply cause a localized activation of downstream Wnt pathway components, resulting in an asymmetric segregation of their activity to the daughters of EMS.

In conclusion, we emphasize that some developmental signals may influence cell fate by directly targeting cytoplasmic components in a responding cell and only in- 
directly regulate gene transcription. As the Wnt pathway is widely conserved, it will be interesting to learn if direct targeting of the cytoskeleton in cells responding to Wnt signaling proves to be of general importance during animal development.

\section{Materials and methods}

\section{Strains and alleles}

Basic C. elegans culturing methods were used as described in Brenner (1974). N2 Bristol was used for the wild-type strain. The mom and pop-1 alleles are recessive, maternal-effect lethal mutations. The mutant strains used were EU340 pop-1(zu189) dpy5(e61) I/hT1 (I;V); him-5(e1490) IV, EU308 mom-1(or10) dpy6(e14) $X / s z T 1(1 ; X)$, EU98 mom-1(or10) lin-2(e1309) $X / s z T 1$ (1;X), EU278 mom-2(or42) V/DnT1 (IV;V), EU95 mom-2(or9) $V / D n T 1(I V ; V)$, EU365 mom-2(or85) V/Dnt1 (IV;V), EU376 rol6(e187) mom-3(or78)/mnC1 II; lin-2(e1309) $X$, EU431 rol1(e91) mom-3(or78) lin-7(e1413)/mnC1 II; him-5(e1490) V, EU309 mom-4(or39) dpy-5(e61) I/hT2 (I;III), and EU452 unc13(e1091) mom-5(zu193) I/hT2 (I;III), EU340 pop-1(zu189) dpy-5(e61) I/hT1 (I;V); him-5(e1490) IV. The JH103 transgenic strain containing the pes-10::GFP construct was provided by G. Seydoux (Seydoux and Fire 1994).

\section{Microscopy and laser ablations}

Light microscopy was performed with a Zeiss Axioscope equipped for Nomarski optics and epifluorescence. Data were either photographed with TechPan 200 film and developed with HC110, or recorded using a Dage-MTI Ve1000 digital camera linked to the Scion Image program run on a Macintosh PowerPC $8100 / 110$. All images were processed using Adobe Photoshop 4.0.

Laser ablations were performed as described previously (Avery and Horvitz 1989). Embryos were fixed and stained with monoclonal antibodies 3NB12 and 5.6 to detect pharyngeal muscle and body wall muscle cells, respectively (Miller et al. 1983; Priess and Thomson 1987; Bowerman et al. 1992).

\section{Blastomere isolation and spindle angle measurements}

Early embryos were obtained by cutting open adult hermaphrodites and blastomeres were isolated and cultured as described previously (Edgar 1995; Shelton and Bowerman 1996). The blastomeres were mechanically separated by repeated mouth pipetting through a glass micropipette. For $\mathrm{P}_{1}$ isolations, sister cells $\mathrm{AB}$ and $\mathrm{P}_{1}$ were separated and $\mathrm{P}_{1}$ was cultured alone. The orientation of the EMS mitotic spindle was determined as illustrated in Figure 1D-axes were designated (black lines) with the plane of contact between EMS and $\mathrm{P}_{2}$ as $90^{\circ}$ and the spindle angle (gray line) was measured relative to these axes. The angle of the EMS spindle was measured when the EMS cytokinetic furrow began to form. At this point in the cell cycle, the mitotic spindle has begun to elongate and the nuclear membrane has broken down. As in intact embryos, the EMS cell cycle was $\sim 15$ min long and $\mathrm{P}_{2}$ divided $\sim 4$ min after EMS. For genetically mosaic partial embryos, wild-type and mutant early EMS and $\mathrm{P}_{2}$ blastomeres were concurrently isolated. EMS and $\mathrm{P}_{2}$ were recombined early in the EMS cell cycle allowing the cells to be in contact for at least 9 min before EMS cytokinesis. For all figures, data from multiple trials were schematically condensed into a single quadrant. Each line on the periphery of the quadrant, representing a data point, is placed at the angle of EMS division.
Identification of C. elegans gsk-3

Degenerate primers were made to regions conserved in known gsk-3 genes but not conserved in other, related Ser/Thr kinases. cDNA was obtained from N2 worms by standard techniques using Trizol reagent (GIBCO-BRL) for RNA isolation and SuperScript II (GIBCO-BRL) for first-strand synthesis. PCR was performed with a MJ-Research PTC-100 thermocycler using Taq polymerase (Perkin Elmer), $1.5 \mathrm{~mm} \mathrm{MgCl}_{2}$, and an annealing temperature of $50^{\circ} \mathrm{C}$. A single $900-\mathrm{bp}$ band was obtained, cloned into pCRII (Invitrogen), and sequenced.

Sequence data were used to design primers for cloning additional cDNA fragments. For all PCR reactions described below, the high-fidelity Pwo polymerase (Boehringer Manheim) was used. The $5^{\prime}$ end of the cDNA was obtained by PCR with a primer to the trans-spliced leader sequence SL1 and internal primer ZW3C. The resulting 556-bp band was cloned into pGem-T (Promega) to yield pJMZWD. A fragment corresponding to bp 461-1227 was obtained by using primers ZW5E and ZW3F and cloned into pGem-T to yield pJMZWF. For sequencing, isolates from at least two independent PCR reactions were obtained, and each isolate was sequenced on both strands. The resulting sequence was used to search a C. elegans EST database, which revealed three cDNA clones corresponding to the gsk-3 gene-yk307d8, yk387f2, and yk451g9. These clones were obtained and analyzed to determine the remaining 3 ' sequence.

\section{RNA-mediated gene interference (RNAi)}

gsk3 RNA was made from plasmid zw3/F1, which contains $\sim 800$ bp of the $5^{\prime}$ coding region in pGEM-T (Promega). The insert was PCR-amplified with SP6 and T7 primers. The PCR product was used to make SP6 and T7 primed RNA, which was mixed to yield double-stranded RNA. apr-1/APC and wrm-1/ arm RNAs were generated from cDNA phage clones using T7 and T3 primers for reverse transcription reactions (apr-1/APC was made from phage clone yk40c12 and wrm-1/arm from yk21a9). ama-1 RNA was made with $\mathrm{T} 7$ primers from plasmid DB19, which contains $\sim 4.7 \mathrm{~kb}$ of the ama-1 coding region in pGEM-7ZF.

RNA was injected at concentrations between 1 and $5 \mu \mathrm{g} / \mathrm{ml}$ into wild-type hermaphrodites, except for ama-1 RNA, which was injected into the transgenic pes-10::GFP strain. After $24 \mathrm{hr}$, injected hermaphrodites were singled out and mated with wildtype males [except for mom-2(RNAi)] to increase the number of progeny. Twelve to $24 \mathrm{hr}$ later, hermaphrodites laying embryos with the appropriate phenotype were used to isolate early embryos.

\section{Transcriptional inhibition}

In embryos from the JH103 strain, GFP fluorescence, driven by the pes-10 promoter, is detectable at the 15-cell stage (after the daughters of EMS have divided), when it comes on in most somatic blastomeres (G. Seydoux, pers. comm.; data not shown). pes-10 expression is detectable at the four-cell stage by RNA in-situ hybridization (Seydoux and Fire 1994; Seydoux et al. 1996). The lag in GFP detection is caused by a delay in translation and the long oxidation step required for GFP fluorescence (G. Seydoux, pers. comm.; Reid and Flynn 1997). GFP fluorescence was checked at the 15 -cell stage as well as $24 \mathrm{hr}$ later. The lack of GFP fluorescence was taken as an indication that inhibition of transcription occurred before transcription of the pes10::GFP construct would have begun at the four-cell stage.

Transcriptional inhibition was achieved using two methods. First, RNA made from ama-1, the gene encoding the largest 
subunit of RNA Pol II was used in RNA-mediated gene interference (Bird and Riddle 1989). ama-1(RNAi) embryos die with $<100$ cells (Powell-Coffman et al. 1996). RNAi was performed on pes-10::GFP hermaphrodites as described above and $\mathrm{P}_{1}$ blastomeres were isolated from embryos collected $36-48 \mathrm{hr}$ after injection. In addition, $\mathrm{P}_{1}$ blastomeres were cultured in media supplemented with $0.5 \mu \mathrm{g} / \mathrm{ml}$ actinomycin D (Sigma, from an $\sim 10 \mathrm{mg} / \mathrm{ml}$ stock in EtOH). These partial embryos arrested as a group of 12 cells, which is the equivalent of the 28-cell stage. With either method alone, GFP expression was not detected in blastomeres and EMS mitotic spindles oriented within the wildtype range (10/10 for ama-1(RNAi) and 10/10 for $0.5 \mu \mathrm{g} / \mathrm{ml}$ actinomycin D). For the experiments shown in Figure $5 \mathrm{~B}, \mathrm{P}_{1}$ blastomeres from ama-1(RNAi) embryos were isolated and allowed to divide in culture media containing $\sim 0.5 \mu \mathrm{g} / \mathrm{ml}$ actinomycin D. The division angle of the EMS spindle was measured as indicated above. Correct EMS spindle orientation was also seen in experiments done using ama-1(RNAi) blastomeres cultured in higher concentrations of actinomycin D [10-20 $\mu \mathrm{g} / \mathrm{ml}$ $(n=6)]$. At the higher concentrations, however, blastomeres sometimes failed to divide even though the EMS spindle oriented normally, apparently attributable to toxic levels of the ethanol solvent (data not shown).

\section{Acknowledgments}

We thank Geraldine Seydoux for the pes-10::GFP strain; Jennifer Knight and Bill Wood for the ama-1 plasmid; Craig Mello and Kevin Fitzgerald for sharing gsk-3 sequence information; and David Miller and Julie Ahringer for antibodies. We are grateful to Yuji Kohara for providing cDNA clones, to the C. elegans Genetic Stock Center [funded by the National Institutes of Health (NIH) National Center for Research Resources] for sending strains, to Matt Thomas and J. Clayton Carter for technical help, and to Chris Doe and Danielle Hamill for helpful advice and comments on the manuscript. We especially thank Cynthia Kenyon for her generosity and support of J.N.M. A.S. and M.M. were supported by training grants from the NIH (GM07413 and HD07348); C.A.S. by a postdoctoral fellowship from the NIH (GM16981); J.N.M. in part by a Howard Hughes Medical Institute predoctoral fellowship, and B.B. by a grant from the NIH (GM49869).

The publication costs of this article were defrayed in part by payment of page charges. This article must therefore be hereby marked 'advertisement' in accordance with 18 USC section 1734 solely to indicate this fact.

\section{Note added in proof}

The GenBank accession no. for gsk-3 is AF159950. A deletion allele of this gene has been isolated by Michael Basson at Axys Pharmaceuticals, Inc. In future publications the gene sgg-1 will replace $g s k-3$.

\section{References}

Avery, L. and H.R. Horvitz. 1989. Pharyngeal pumping continues after laser killing of the pharyngeal nervous system of $C$. elegans. Neuron 3: 473-485.

Axelrod, J.D., J.R. Miller, J.M. Shulman, R.T. Moon, and N. Perrimon. 1998. Differential recruitment of Dishevelled provides signaling specificity in the planar cell polarity and wingless signaling pathways. Genes \& Dev. 12: 2610-2622.

Bird, D.M. and D.L. Riddle. 1989. Molecular cloning and se- quencing of ama-1, the gene encoding the largest subunit of Caenorhabditis elegans RNA polymerase II. Mol. Cell. Biol. 9: 4119-4130.

Boutros, M., N. Paricio, D.I. Strutt, and M. Mlodzik. 1998. Dishevelled activates JNK and discriminates between JNK pathways in planar polarity and wingless signaling. Cell 94: 109-118.

Bowerman, B., B.A. Eaton, and J.R. Priess. 1992. skn-1, a maternally expressed gene required to specify the fate of ventral blastomeres in the early C. elegans embryo. Cell 68: 10611075.

Boyle, W.J., T. Smeal, L.H. Defize, P. Angel, J.R. Woodgett, M. Karin, and T. Hunter. 1991. Activation of protein kinase C decreases phosphorylation of c-Jun at sites that negatively regulate its DNA-binding activity. Cell 64: 573-584.

Brannon, M., M. Gomperts, L. Sumoy, R.T. Moon, and D. Kimelman. 1997. A beta-catenin/XTcf-3 complex binds to the siamois promoter to regulate dorsal axis specification in Xenopus. Genes \& Dev. 11: 2359-2370.

Brenner, S. 1974. The genetics of Caenorhabditis elegans. Genetics 77: 71-94.

Cadigan, K.M. and R. Nusse. 1997. Wnt signaling: A common theme in animal development. Genes \& Dev. 11:32863305.

Edgar, L.G. 1995. Blastomere culture and analysis. In Caenorhabditis elegans: Modern biological analysis of an organism (ed. H.F. Epstein and D.C. Shakes), pp. 303-321. Academic Press, San Diego, CA.

Edgar, L.G., N. Wolf, and W.B. Wood. 1994. Early transcription in Caenorhabditis elegans embryos. Development 120: 443451.

Fire, A., S. Xu, M.K. Montgomery, S.A. Kostas, S.E. Driver, and C.C. Mello. 1998. Potent and specific genetic interference by double-stranded RNA in Caenorhabditis elegans. Nature 391: 806-811.

Goldstein, B. 1992. Induction of gut in Caenorhabditis elegans embryos. Nature 357: 255-257.

- 1993. Establishment of gut fate in the E lineage of $C$. elegans: The roles of lineage-dependent mechanisms and cell interactions. Development 118: 1267-1277.

- 1995a. An analysis of the response to gut induction in the C. elegans embryo. Development 121: 1227-1236.

- 1995b. Cell contacts orient some cell division axes in the Caenorhabditis elegans embryo. J. Cell Biol. 129: 10711080.

Goldstein, B., S.N. Hird, and J.G. White. 1993. Cell polarity in early C. elegans development. Development Suppl. 279-287.

Hawkins, N. and G. Garriga. 1999. Asymmetric cell division: From A to Z. Genes \& Dev. 12: 3625-3638.

He, X., J.-P. Saint-Jeannet, J.R. Woodgett, H.E. Varmus, and I.B. Dawid. 1995. Glycogen synthase kinase-3 and dorsoventral patterning in Xenopus embryos. Nature 374: 617-622.

Hyman, A.A. 1989. Centrosome movement in the early divisions of Caenorhabditis elegans: A cortical site determining centrosome position. J. Cell Biol. 109: 1185-1193.

Hyman, A.A. and J.G. White. 1987. Determination of cell division axes in the early embryogenesis of Caenorhabditis elegans. J. Cell Biol. 105: 2123-2135.

Ikeda, S., S. Kishida, H. Yamamoto, H. Murai, S. Koyama, and A. Kikuchi. 1998. Axin, a negative regulator of the Wnt signaling pathway, forms a complex with GSK- $3 \beta$ and $\beta$-catenin and promotes GSK- $3 \beta$-dependent phosphorylation of $\beta$-catenin. EMBO J. 17: 1371-1384.

Kemphues, K. and S. Strome. 1997. Fertilization and establishment of polarity in the embryo. In Caenorhabditis elegans II (ed. D.L. Riddle, T. Blumenthal, B.J. Meyer and J.R. Priess), 
pp. 335-360. Cold Spring Harbor Laboratory Press, Cold Spring Harbor, NY.

Lin, R., S. Thompson, and J.R. Priess. 1995. pop-1 encodes an HMG box protein required for the specification of a mesoderm precursor in early C. elegans embryos. Cell 83: 599609.

Lin, R., R.J. Hill, and J.R. Priess. 1998. POP-1 and anterior-posterior fate decisions in C. elegans embryos. Cell 92: 229-239.

Lu, B., L.Y. Jan, and Y. N. Jan. 1998. Asymmetric cell division: Lessons from flies and worms. Curr. Opin. Genet. Dev. 8: 392-399.

Malathi, K., Y. Xiao, and A.P. Mitchell. 1997. Interaction of yeast repressor-activator protein Ume6p with glycogen synthase kinase 3 homolog Rim11p. Mol. Cell Biol. 17: 7230 7236.

Mandel, S., K. Robzyk, and Y. Kassir. 1994. IME1 gene encodes a transcription factor which is required to induce meiosis in Saccharomyces cerevisiae. Dev. Genet. 15: 139-147.

Miller, D.M., III, I. Ortiz, G.C. Berliner, and H.F. Epstein. 1983. Differential localization of two myosins within nematode thick filaments. Cell 34: 477-490.

Miller, J.R. and R.T. Moon. 1997. Analysis of the signaling activities of localization mutants of $\beta$-catenin during axis specification in Xenopus. J. Cell Biol. 139: 229-243.

Powell-Coffman, J.A., J. Knight, and W.B. Wood. 1996. Onset of C. elegans gastrulation is blocked by inhibition of embryonic transcription with an RNA polymerase antisense RNA. Dev. Biol. 178: 472-483.

Priess, J.R. and J.N. Thomson. 1987. Cellular interactions in early C. elegans embryos. Cell 48: 241-250.

Reid, B.G. and G.C. Flynn. 1997. Chromophore formation in green fluorescent protein. Biochemistry 36: 6786-6791.

Rocheleau, C.E., W.D. Downs, R. Lin, C. Wittmann, Y. Bei, Y.H. Cha, M. Ali, J.R. Priess, and C.C. Mello. 1997. Wnt signaling and an APC-related gene specify endoderm in early C. elegans embryos. Cell 90: 707-716.

Rubin-Bejerano, I., S. Mandel, K. Robzyk, and Y. Kassir. 1996. Induction of meiosis in Saccharomyces cerevisiae depends on conversion of the transcriptional repressor Ume6 to a positive regulator by its regulated association with the transcriptional activator Ime1. Mol. Cell Biol. 16: 2518-2526.

Rubinfeld, B., I. Albert, E. Porfiri, C. Fiol, S. Munemitsu, and P. Polakis. 1996. Binding of GSK3 $\beta$ to the APC- $\beta$-catenin complex and regulation of complex assembly. Science 272: 1023 1026.

Schierenberg, E. 1987. Reversal of cellular polarity and early cell-cell interactions in the embryo of Caenorhabditis elegans. Dev. Biol. 122: 452-463.

Seydoux, G. and A. Fire. 1994. Soma-germline asymmetry in the distributions of embryonic RNAs in Caenorhabditis elegans. Development 120: 2823-2834.

Seydoux, G., C.C. Mello, J. Pettitt, W.B. Wood, J.R. Priess, and A. Fire. 1996. Repression of gene expression in the embryonic germ lineage of C. elegans. Nature 382: 713-716.

Shelton, C.A. and B. Bowerman. 1996. Time-dependent responses to $g 1 p$-1-mediated inductions in early C. elegans embryos. Development 122: 2043-2050.

Siegfried, E., T.-B. Chou, and N. Perrimon. 1992. wingless signaling acts through zeste-white 3, the Drosophila homolog of glycogen synthase kinase-3, to regulate engrailed and establish cell fate. Cell 71: 1167-1179.

Strich, R., R.T. Surosky, C. Steber, E. Dubois, F. Messenguy, and R.E. Esposito. 1994. UME6 is a key regulator of nitrogen repression and meiotic development. Genes \& Dev. 8: $796-$ 810.

Sulston, J.E., E. Schierenberg, J.G. White, and J.N. Thomson.
1983. The embryonic cell lineage of the nematode Caenorhabditis elegans. Dev. Biol. 100: 64-110.

Thorpe, C.J., A. Schlesinger, J.C. Carter, and B. Bowerman. 1997. Wnt signaling polarizes an early C. elegans blastomere to distinguish endoderm from mesoderm. Cell 90: 695-705.

Waddle, J.A., J.A. Cooper, and R.H. Waterston. 1994. Transient localized accumulation of actin in Caenorhabditis elegans blastomeres with oriented asymmetric divisions. Development 120: 2317-2328.

Wagner, U., J. Brownlees, N.G. Irving, F.R. Lucas, P.C. Salinas, and C.C.J. Miller. 1997. Overexpression of the mouse dishevelled-1 protein inhibits GSK-3 $\beta$-mediated phosphorylation of tau in transfected mammalian cells. FEBS Lett. 411: 369-372.

Wong, L.L. and P.N. Adler. 1993. Tissue polarity genes of Drosophila regulate the subcellular location for prehair initiation in pupal wing cells. J. Cell Biol. 123: 209-221. 


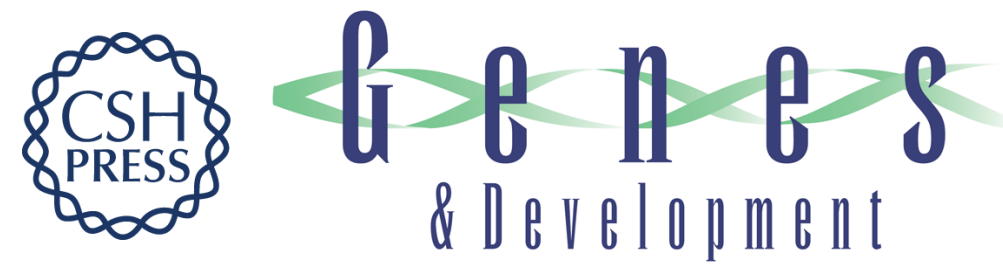

\section{Wnt pathway components orient a mitotic spindle in the early Caenorhabditis elegans embryo without requiring gene transcription in the responding cell}

Ann Schlesinger, Christopher A. Shelton, Julin N. Maloof, et al.

Genes Dev. 1999, 13:

References

This article cites 44 articles, 22 of which can be accessed free at:

http://genesdev.cshlp.org/content/13/15/2028.full.html\#ref-list-1

License

Email Alerting

Service

Receive free email alerts when new articles cite this article - sign up in the box at the top right corner of the article or click here.

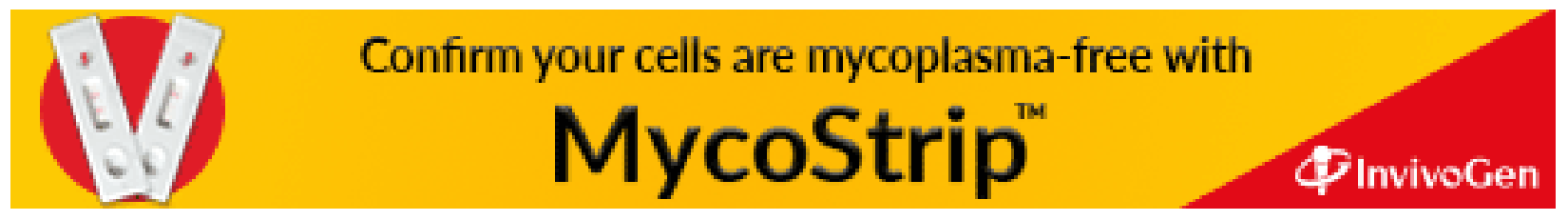

